\title{
Customizing planetary gear trains for human limb assistance and replication
}

\author{
Pablo Lopez Garcia ${ }^{1,{ }^{*}}$, Stein Crispel $^{1}$, Tom Verstraten $^{1}$, Elias Saerens ${ }^{1}$, Bram Vanderborght $^{1}$, and Dirk Lefeber ${ }^{1}$ \\ ${ }^{1}$ BRUBOTICS, Vrije Universiteit Brussel and Flanders Make, Brussels, Belgium
}

\begin{abstract}
Human-Robot Interaction (HRI) devices are becoming increasingly customary to assist humans, both in industrial and clinical environments. Traditional robotic gearbox technologies, like cycloid reducers or Harmonic Drives (C), perform well to solve the need for extreme precision and high speed of industrial robots, but when used in HRI devices they are usually forced above their limits, ultimately restraining the performance and the widespread use of such devices. A closer analysis of the specific needs behind the actuation for human limb assistance and replication highlights the differences with traditional robotic applications. Matching those needs with the main characteristics of different robotic gearboxes displays the limitations of traditional robotic transmissions, but it also unveils the potential of a virtually unexploited kind of planetary gear trains. This paper presents the prototype and associated first test results of the Wolfrom alpha-I concept. This novel concept demonstrates the application potential of Wolfrom-based planetary gear trains in HRI actuation.
\end{abstract}

\section{Introduction}

Human-Robot Interaction (HRI) devices, like exoskeletons or actuated prostheses, are increasingly common in manufacturing environments and clinical applications [1,2]. Yet, the performance of such devices is currently strongly limited by the confined possibilities of their actuators in terms of mass, compactness or efficiency [3].

To improve the wearability and autonomy of these robotic aids, the drivetrains and the batteries used to power them must achieve better efficiencies and reduce their weight, enabling higher specific torques $[4,5]$. Selecting suitable transmission technologies is the usual engineering answer to such challenges. In industrial robotics, Harmonic Drives $\mathbb{C}$, cycloidal reducers and, to a lesser degree, planetary gear trains (PGTs), have established themselves as the dominant transmission technologies [6].

The structure of the paper is as follows. First, in chapter 2 we analyse the needs of HRI actuation in the example of the ankle-prostheses actuation. We then demonstrate how these needs force current robotic technologies beyond their possibilities, and how the extreme versatility of planetary gear trains can be used to design transmissions better aimed at the needs of HRI actuation. Chapter 3 describes how the authors have used this approach to build a concept prototype, for which the first experimental results are also presented. Finally, the conclusions and the list of references close the contents of this paper.

\section{Case study human limb assistance and replication}

\subsection{Background}

Human muscles are not characterized by very high efficiencies or high specific power characteristics. However, they can provide impressive specific forces and they are enormously versatile, to assist the highly dynamic biomechanical actuation, with fast and continuously changing speeds and fast variations of the mechanical impedance (ratio between force and velocity) within a very broad range of values [7].

The knee, ankle and hip joints play a fundamental role during human locomotion [8]. Active HRI systems designed to assist locomotion, mechanically support or replace these joints, and they must therefore be able to follow their respective working cycles. Figs. 1 and 2 (left) schematically present some examples of these cycles for a human ankle. For a complete walking cycle of the ankle, which lasts around 1 second for normal walking speed, the curves show relatively low speeds, surprisingly high peak torques, and frequent periods of negative work. During these negative-work periods, energy is reverted into our joints and stored into the (elastic) tendons and ligaments in our lower limbs, for later usage during the gait process. Hence, to cope with the elevated maximum torques, heavy and bulky actuators are needed. And this also means that, during most of the time, these heavy actuators will be operating

\footnotetext{
Corresponding author: plopezga@vub.be
} 
at very low and even negative torques, far from their optimal efficiency [9].

This situation can be extrapolated to most HRI applications. Whereas, in most traditional industrial robotics and conventional industrial applications, motors are optimized for constant speed and continuous power dissipation, in HRI almost-ballistic trajectories are the rule [10]. This, combined with the central role of efficiency in these applications, represents a challenge to select the most suitable actuation system [9].

As previously mentioned, adding transmissions to the actuation system is the usual engineering response to this sort of challenges. Particularly for this case, variable transmissions with multiple reduction ratios, each optimized for a different region of the operating cycle of the actuator, are the natural choice. Unfortunately, variable transmissions tend to be heavy and bulky, complicating their efficient integration in HRI applications. Although some research exists in this direction [11], no real breakthrough has yet been attained.
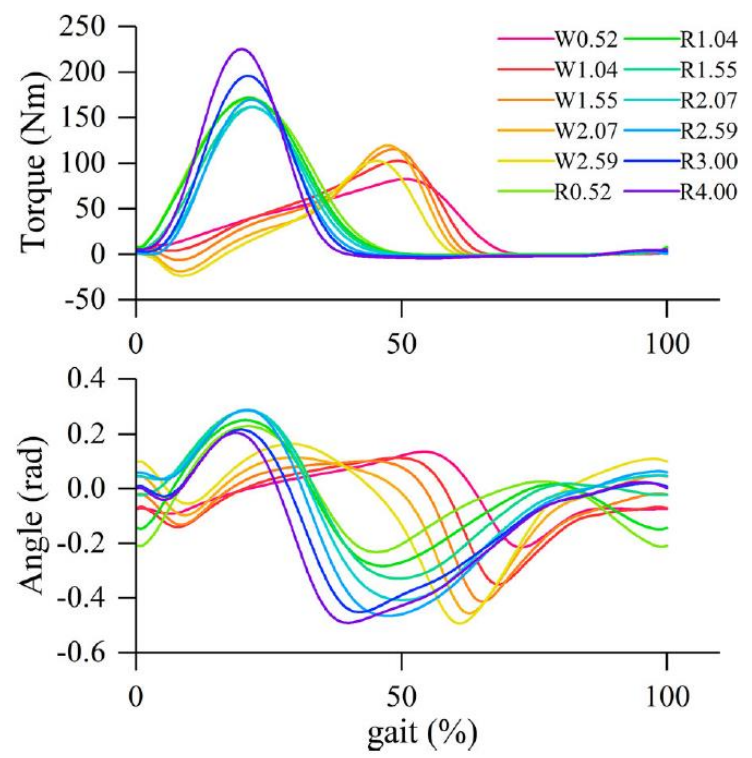

Fig. 1. Biomechanical (torque and angle) evolution of the human ankle during gait transition at different walking (W) and running $(\mathrm{R})$ speeds for a $75 \mathrm{~kg}$. person [12].

An approach worth mentioning, due to its relevance in the field of HRI, is the attempt to exploit the cyclic character of HRI actuation to reduce the actuator size and weight, by means of incorporating compliance to the actuation system, mimicking the ability of our limb to store mechanical energy. Unfortunately, this approach has also a number of drawbacks for which [13] provides further detail, and which in practice limit the extent to which this solution can be used to reduce the size and mass of the actuators.

In conclusion, when designing a lower limb actuation system, robotic engineers tend to conceive first a mechanical system incorporating a limited amount of compliance, to reduce the maximum power that the actuator must provide. They typically select then the lightest suitable actuator - usually an electrical motor - according to this remaining maximum power demand, and select also a high-gear ratio transmission system (generally between 1:200 and 1:500) to amplify the torque capabilities of the actuator and comply with the high max-torque needs, taking advantage at the same time of the low maximum speeds required.

Additionally, ergonomic aspects must be considered when incorporating robotic actuation systems to wearable robotic devices. For actuation systems, this translates into favouring disc-shaped solutions - with higher diameters but limited axial dimensions - in order to minimize interference with the natural movements of the subject. The experience of our lab in this field indicates that gearboxes with diameters below $12 \mathrm{~cm}$ and axial dimensions below $6 \mathrm{~cm}$ are particularly favourable for efficient ergonomic integration in lower and upper limbs.

\subsection{Traditional industrial-robotics transmissions}

Industrial robots have an extreme need for high precision, required to be able to perform their tasks. This need has been central for the current usage ranking of robotic gearbox technologies.

\subsubsection{Strain wave gears}

One of the most used technologies in robotics is the Harmonic Drive (C) (HD), a system that takes advantage of the ability of strain wave gears with multiple teeth contact to provide high gear ratios in a very compact shape, and with extreme positioning accuracy. In their pancake format, HD's are compatible with larger diameters and limited axial dimensions, making them particularly suitable for wearable devices. In industrial manipulators, they are normally used in the joints closer to the end effectors, where their limited overload ability is not an obstacle and their low mass represents an important advantage to optimize the manipulator's bandwidth.

Unfortunately, the efficiency of the HD is not very high, and it shows a strong dependency on the operating conditions [14]. This makes it especially challenging to optimize efficiency in systems actuated with electrical motors and variable operating conditions [15].
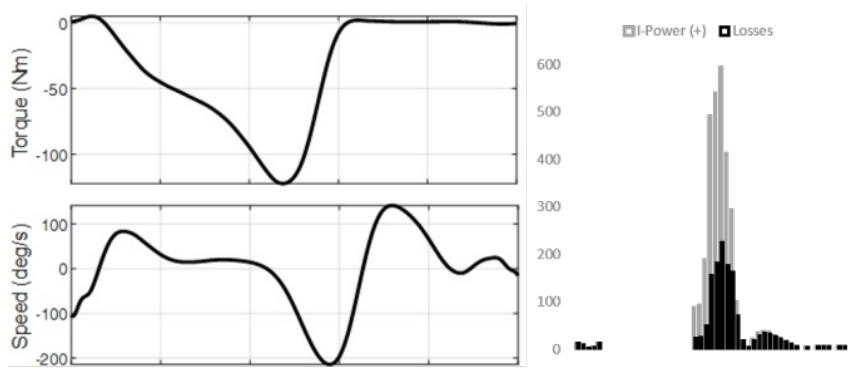

Fig. 2. (Left) Operational Curve of an ankle actuator for a $75 \mathrm{~kg}$ person, walking at normal speed, and (right) simulated power losses (black) versus input power (grey) in a CSD 252A/100 Harmonic Drive following an ankle actuation cycle, calculated using the manufacturer's efficiency model [14]. The resulting average transmission efficiency was $56.6 \%$. 
According to our own simulations and tests for the example of lower limb actuation (Fig.2), this results in average gearbox efficiencies close to $50 \%$, and therefore in the need to almost duplicate of the size and weight of the battery.

Another drawback of the HD is that its gear ratio is practically limited to maximum values up to $1: 160$ for a single stage configuration. HRI engineers therefore tend to combine these devices with crank- or beltmechanisms that provide the remaining gain required to achieve the typical 1:200 to 1:500 overall ratios needed.

\subsubsection{Cycloidal reducers}

Cycloidal reducers are based on the same fundamental principle as the strain wave gears, the small-toothdifference, internal contact type gear train. They use rolling contacts instead of teeth to transfer torque and speed. They can provide as well very high gear ratios in a few stages and show an ergonomically compatible disc-shape [10].

Its efficiency is higher than for $\mathrm{HD}$, thanks to the rolling contacts instead of teeth meshing, but highly dependent also on the manufacturing precision. The major drawback is that they are not as compact or light as a HD: they tend to be heavier and bulkier, and they typically need an additional stage to compensate for the inherent unbalance resulting from their operating principle.

In industrial robotics, these devices are typically used in combination with a parallel shaft gear train input, since cycloids are vulnerable to high input speeds. They find predominantly application in the less distal joints of industrial manipulators, far from the end-effectors, where their high resilience against overload becomes a major advantage, and their higher weight and volume is less relevant.

Analog to the HD, cycloid reducers are practically limited to maximum gear ratios which for reduced sizes are typically in the range of 1:30 to 1:50, and they also need to incorporate other auxiliary elements providing additional gain.

In HRI, they are scarcely used due to their high mass, limited gear ratio and manufacturing complexity, although some researchers have included them in their designs $[10,16]$.

\subsubsection{Planetary gear trains}

Planetary Gear Trains can achieve very high efficiencies, which are not strongly dependent on the operating conditions, and their ability to split power between the different planets makes them very compact. Yet, they have progressively been displaced from the joints of industrial robots, mainly due to their limited ability to reduce backlash, which results in lower positioning precision for the robotic manipulator.

The topology predominantly used in industry is the planetary arrangement (with a grounded annular ring) for which the maximum practicable gear ratios per stage are limited to around 1:10 (typical values are usually below
1:8). This means that either auxiliary mechanical elements need to be incorporated, or multiple planetary stages must be concatenated to obtain the high gear ratios, leading in both cases to heavy and bulky units.

Additionally, the gearheads commercially available tend to reproduce the cylindrical shape characteristic of the electrical motors, with long axial and limited radial dimensions. As we have previously seen, this shape is not particularly advantageous for an ergonomic integration into wearable robotic devices.

\subsection{Other planetary gear train configurations}

Although the planetary arrangement with cylindrical shape is by far the most widespread configuration of planetary gear trains, due to its evident advantages for most industrial applications, planetary gear trains are extremely versatile in terms of shape and configurations.

Examples of this versatility are e.g. the Ravigneaux or the Simpson PGTs [17], which find a wide application among others in automotive automatic transmissions. Another interesting configuration invented over a hundred years ago, and with the ability to achieve very high gear ratios (also above 1:400) in a very compact shape, is the Wolfrom PGT [17] shown in Fig.3.

Due to the inherent low efficiencies that immediately follow from high gear ratios in a Wolfrom PGT, its use has historically been very marginal and restricted mainly to low-speed, low-power applications [18].

For the Wolfrom PGT, where one annular ring is used as the system's output, deviating from the commercially standard cylinder shape to disc-shaped planetary configurations brings advantages in terms of the system maximum torque: it enables larger lever arms, and the possibility to use higher teeth modules while maintaining the same number of teeth.

\section{A Wolfrom reducer prototype}

In Wolfrom PGTs (see Fig.3), the two meshing contacts of the (compound) planet gearwheels with both the grounded and the output annular rings are subjected simultaneously to high torque levels and high relative speeds. The product of these two elements is termed "virtual" power and it is proportional to the contact losses generated in the meshing contact [19]. $\mathrm{n}$ the two meshing contacts between the planet gears and the ring gears, this "virtual" power is at least an order higher than the input power of the system, especially when high gear ratios are used. This high virtual power is the driving force behind the characteristically low efficiency of a Wolfrom PGT. 


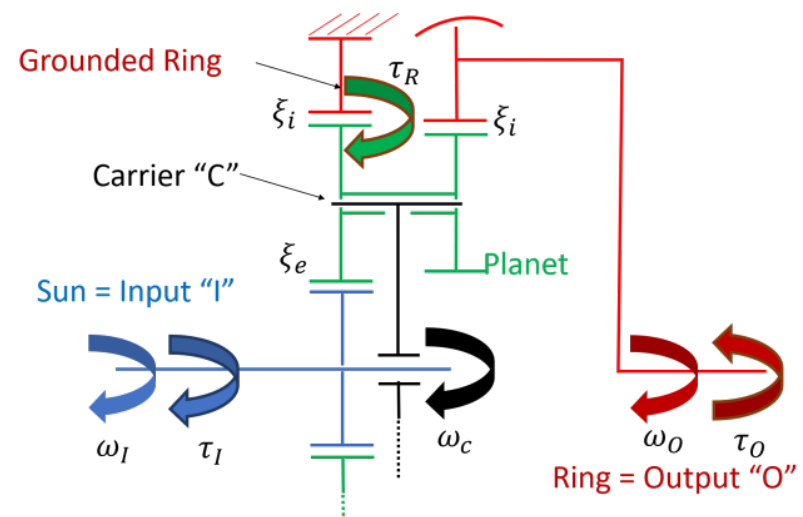

Fig. 3. A Wolfrom planetary gear train indicating its internal and external meshings, and the respective loss coefficients $\xi \mathbf{i}$, $\xi \mathbf{e}$ given by $\eta_{\mathrm{i}}=1-\xi \mathrm{i}$ and $\eta_{\mathrm{e}}=1-\xi \mathrm{e}$, where $\eta=$ meshing efficiency. $\tau$ represents the torques, and $\omega$ the rotational speeds.

The possibility to manufacture gears with increasingly high precision has paved the way for the use of low-loss gears: balancing approach and recess paths, while simultaneously optimizing (minimizing) other parameters like contact ratio, pressure angle or teeth module, allows to improve the meshing efficiencies of involute gears by up to $70 \%$ [20]. Applying these advances to Wolfrom PGTs could make high gear ratios compatible with maintaining good efficiencies over a very broad range of operating conditions, resulting in a transmission extremely well suited for HRI actuation.

\subsection{The Alpha-I concept prototype}

With the aid of a Failure Mode and Effect Analysis (FMEA) some of the main challenges in designing a Wolfrom reducer gearbox for HRI actuation were identified [7]. These challenges include among others (i) making reduced teeth widths compatible with maintaining an adequate meshing alignment while avoiding unwanted contacts between neighbouring meshing's, and (ii) validating the effect of improved meshing efficiency on the overall gearbox efficiency.

Due to the particular importance of efficiency for the Wolfrom configuration, and to the need to deviate from standard teeth widths to optimize the disc-shape of our gear train [21], we decided to focus the design of our first concept prototype in the demonstration of the viability of these two particular aspects. Other important aspects, including for example suitable strategies (floating or flexible elements) to improve the load distribution between the different planets [22], or the feasibility of precise phase shift between the two planetary gearwheels, were deemed less relevant for a preliminary validation and purposely not integrated in our design.

Measuring efficiency in planetary gear trains is very challenging because of the high sensing accuracy demanded to measure the typically extremely high efficiencies of these devices. Manufacturing the different elements of the prototype such that small deviations (alignment, precision, etc.) did not perturbate the efficiency analysis represented a first important obstacle, especially with a restricted budget. And approaching serial openly was also not possibly, due to Intellectual Property Rights issues.

Mainly for these reasons we decided to limit the manufacturing precision challenge, duplicating the size $(2: 1)$ of our prototype. For the same reasons, it was decided to use laser sintered (SLS) printed Polyamide (PA12) gears instead of steel gears. This results in lower meshing efficiency, which actually simplifies the measurements and enables a simpler traceability of the impact of the meshing efficiency in the overall system efficiency.

Selecting an adequate gear ratio was also an important design decision. One of the advantages of the Wolfrom PGT is the possibility to provide totality of the typical gain levels required by HRI systems, without the need to incorporate additional crank- of belt systems. Yet, we selected a gear ratio close to 1:100 for our design, since that was sufficient to demonstrate the functionality and it was also more suitable to compare the prototype with other existing gearbox technologies.

The teeth geometry of the two meshings between the planetary gears and the two ring gearwheels was optimized for low loss by means of limiting and balancing the approach and recess contact ratios. A contact ratio in the vicinity of 1.5 was targeted for both meshings, enough to provide a substantial efficiency improvement (see Fig. 4), while maintaining also sufficient teeth overlap to assure a continuous contact condition.

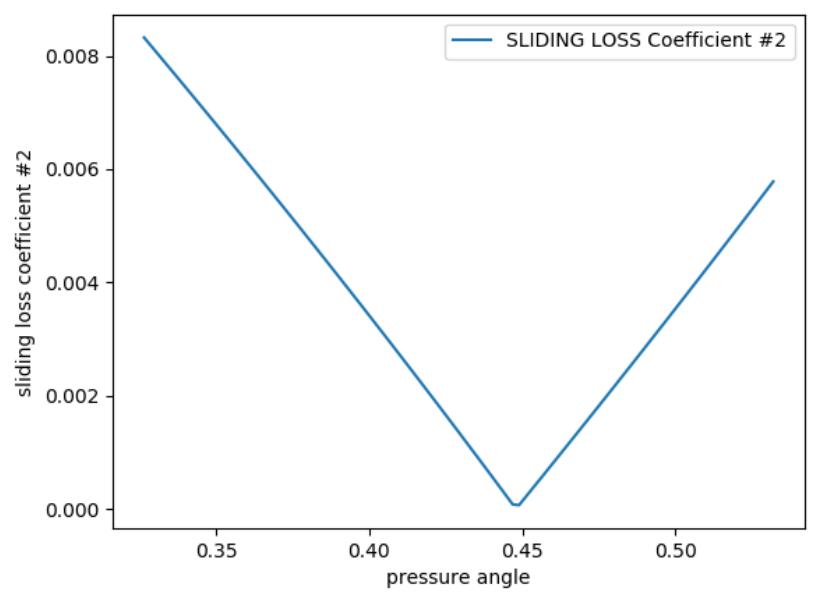

Fig. 4. Evolution of the theoretical Sliding Loss Coefficient $\xi \mathrm{i}=1-\eta \mathrm{i}$, measured along the line of contact, for the internal meshing between the first planetary gearwheel, and the first annular ring, assuming a usual friction coefficient value for gear steels (0.1) and after the optimization of the contact ratio.

Due to the lower impact of the external meshing on the overall system efficiency, a commercially available KHK SS2-12 gearwheel made from S45C steel was used as sun gear. NSK- ball bearings were placed between the carrier and the output annular ring, as well as between the central shaft and both annular rings and the carrier. For the carrier to planetary sets, we decided to fix the pins (pressing-fit) directly to the carrier, and to 
incorporate bushing bearings to allow the planetary gearwheels to freely rotate around the carrier pins.

Finally, a module of $2 \mathrm{~mm}$ was selected for the Alpha-I prototype as adequate teeth size. This corresponds to a module of $1 \mathrm{~mm}$ in the reference gearbox design in steel, suitable for an expected maxtorque around $80 \mathrm{Nm}$ [23].

Fig. 5 and 6 provide some pictures of the aspect and resulting dimensions of our Alpha-I concept prototype, while Table 1 presents its main design parameters.

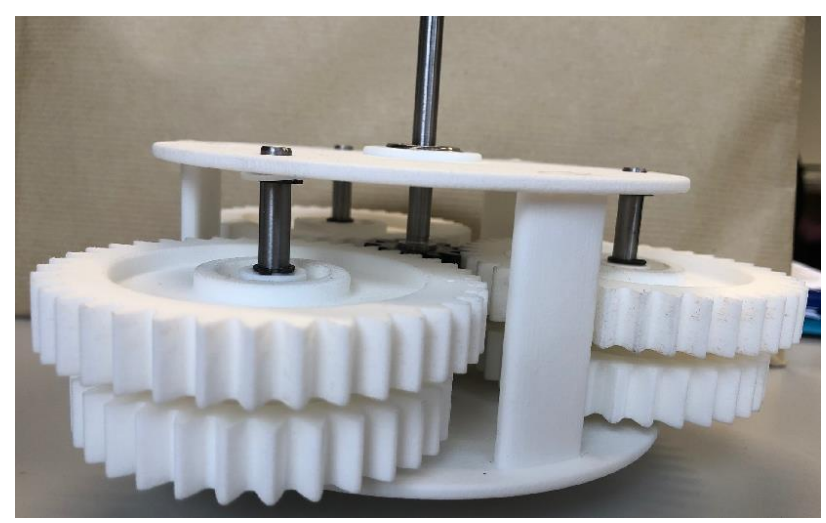

Fig. 5. Carrier Assembly of the Alpha-I prototype.

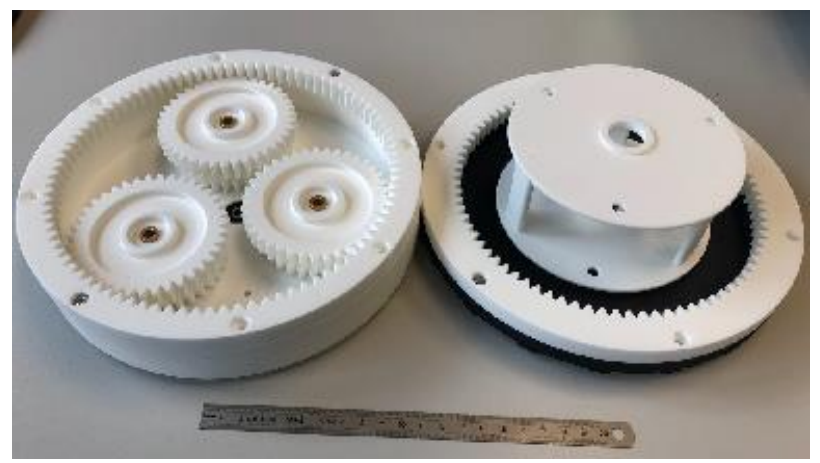

Fig. 6. Set of Wolfrom gearwheels and carrier cage in Alpha-I prototype.

Table 1. Main design parameters of the Alpha-I prototype.

\begin{tabular}{|c|c|}
\hline Element & Style \\
\hline Prototype scale & $2: 1$ \\
\hline Teeth module(s) & $2 \mathrm{~mm}\left(2,1 \mathrm{~mm}\right.$ for $2^{\text {nd }}$ stage $)$ \\
\hline Number of Teeth & $\begin{array}{l}12(\mathbf{S}, \text { sun wheel }), 90(\mathbf{R}, \\
\text { grounded annular ring }), 81(\mathbf{O}, \\
\text { output annular ring }), 39\left(\mathbf{P 1}, 1^{\text {st }}\right. \\
\text { planet wheel }), 32\left(\mathbf{P 2}, 2^{\text {nd }} \text { planet }\right. \\
\text { wheel })\end{array}$ \\
\hline Gear Ratio & $1: 96.2$ \\
\hline Contact Ratios & $\begin{array}{l}1.48 \text { (grounded annular ring) and } \\
1.57 \text { (output annular ring) }\end{array}$ \\
\hline Outer Diameter & $210 \mathrm{~mm}$ \\
\hline Axial Width & $80 \mathrm{~mm}$ \\
\hline Materials & $\begin{array}{l}\text { S45C (sun gearwheel) and SLS- } \\
\text { printed PA12 (rest) }\end{array}$ \\
\hline
\end{tabular}

\subsection{Experimental results}

Representative testing of high gear ratio gearboxes for applications with fluctuating operating conditions introduces multiple challenges to the required test rigs [24]. In order to perform basic testing in our prototype, without the availability of suitable, high-accurate test rigs, we decided to simplify the measurements and take advantage of the quasi-ballistic character of the operating trajectory. The gearbox was actuated with an EC40 Maxon and tested in a set-up where an eccentric load had to be first lifted and then lowered. The test was done with a frequency of $0.125 \mathrm{~Hz}$, slow enough to limit dynamic effects but sufficiently close to the usual frequencies in lower limb actuation [25]. The set-up is described in more detail in Fig.7
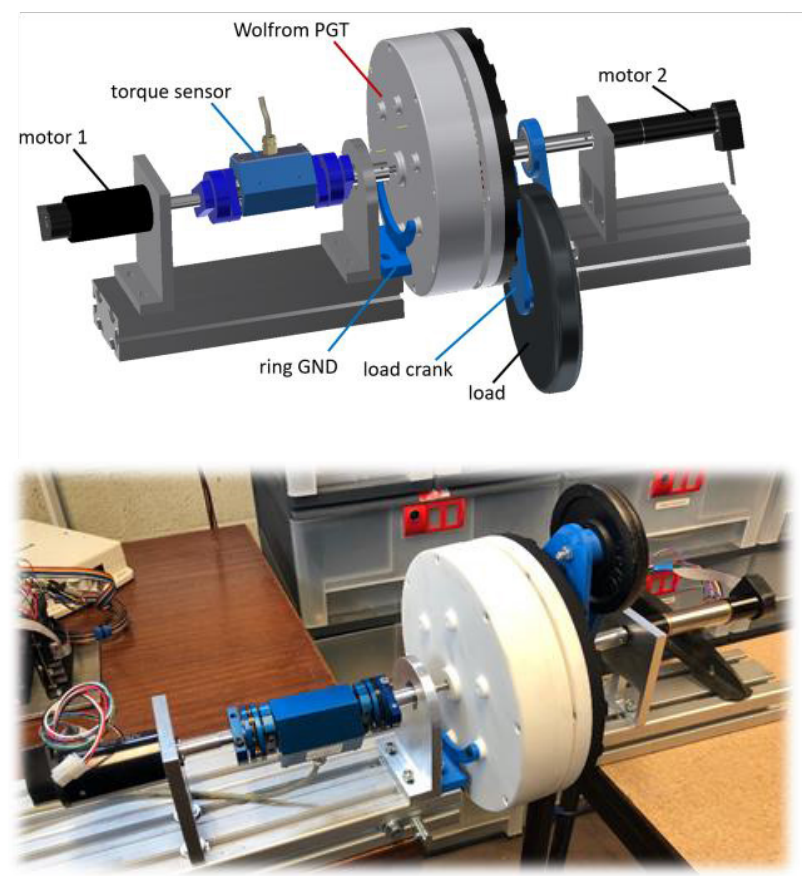

Fig. 7. Test-Set Up: a $120 \mathrm{~W}$ motor (Maxon EC40 387601 , motor 1) with a stall toque of $0,74 \mathrm{Nm}$ is used to drive the sun gearwheel of the Wolfrom gearbox in which the first ring is grounded (ring GND) and the second ring is attached to a load crank driving an eccentric load (weight on lever arm). A torque sensor is incorporated between the input motor and the PGT. Finally, the carrier of the PGT is attached to a $120 \mathrm{~W}$ motor (Maxon EC-4pole 22 311536, motor 2) incorporating a 109:1 gearbox (Maxon GP22 370784) and a brake (Maxon AB20 301212), enabling the operation of the PGT in its basic configuration (carrier grounded). Although both motors incorporate Hall sensing capabilities, suitable Maxon HEDL optical encoders have also been adjusted to each of the motors, to enable a more reliable speed measurement.

With the aid of this simple test set-up the gear ratio of our Wolfrom gearbox could be validated. Additionally, it was also possible to perform preliminary efficiency measurements and to make a first range validation of the scaling effect of the meshing efficiency in a Wolfrom gearbox. The test battery presented in Table 2 was executed for this purpose. 
Table 2. Test Battery.

\begin{tabular}{|c|c|}
\hline Test & Description \\
\hline Fixed carrier, unloaded & $\begin{array}{c}\text { Input speed }=60 \mathrm{rpm} \\
\text { Output speed }=7.3 \mathrm{rpm} \\
\text { No Load }\end{array}$ \\
\hline Fixed carrier, loaded & $\begin{array}{c}\text { Input speed }=60 \mathrm{rpm} \\
\text { Output speed }=7.3 \mathrm{rpm} \\
\text { Mass-Load }=0.5 \mathrm{~kg} \\
\text { Lever arm } 120 \mathrm{~cm}\end{array}$ \\
\hline Fixed ring, unloaded & $\begin{array}{c}\text { Input speed }=679 \mathrm{rpm} \\
\text { Output speed }=7.1 \mathrm{rpm} \\
\text { No Load }\end{array}$ \\
\hline Fixed ring, loaded & $\begin{array}{c}\text { Input speed }=679 \mathrm{rpm} \\
\text { Output speed }=7.1 \mathrm{rpm} \\
\text { Mass-Load }=0.5 \mathrm{~kg} \\
\text { Lever arm } 120 \mathrm{~cm}\end{array}$ \\
\hline
\end{tabular}

\subsubsection{Fixed carrier}

The brake of the motor 2, fixed to the carrier, is used to ground this element and let the gearbox operate as a quasi-planetary system, with a gear ratio that corresponds to the following expression $(\mathrm{Z}=$ number of teeth):

$$
i=-Z_{P 1} Z_{O} / Z_{S} Z_{P 2}=-8.23
$$

Fig. 8 presents the results of the measurements of the current input during operation with a mass-load of $0.5 \mathrm{~kg}$ and without any load, with the operating conditions provided in Table 2. The driven current and the torque provided by a motor can be related with the aid of the torque constant of the motor $\left(k_{t}=76.4 \mathrm{mNm} / \mathrm{A}\right.$ for motor 1 , according the datasheet), using the following equation including damping losses $v \omega$ :

$$
k_{t} I=\boldsymbol{\tau}+v \omega
$$

where $I$ is the driven current, $\boldsymbol{\tau}$ is the motor torque and $\omega$ is the motor speed, which is approximately constant in this case. Subtracting the driven currents in the loaded and unloaded cases, to cancel the damping losses, and multiplying then by the torque constant, the torque required to spin the $0,5 \mathrm{~kg}$ load in these conditions can be obtained, as presented in Fig. 8.

According to this calculation, the peak of the motor torque difference $(75 \mathrm{mNm})$ corresponds to the point of maximum output torque of:

$$
\boldsymbol{\tau} \text { out }=0.5 \mathrm{~kg} * 0.12 \mathrm{~m} * 9,8 \mathrm{~m} / \mathrm{s}^{2}=588 \mathrm{mNm}
$$

And the corresponding torque ratio:

$$
t=\boldsymbol{\tau} \text { out } / \boldsymbol{\tau} \text { in }=588 / 73.9=7.96
$$

This enables us to calculate the efficiency of the quasi-planetary configuration to spin the $0.5 \mathrm{~kg}$ mass at the peak torque point as:

$$
\eta c=7.96 / 8.23=96.7 \%
$$

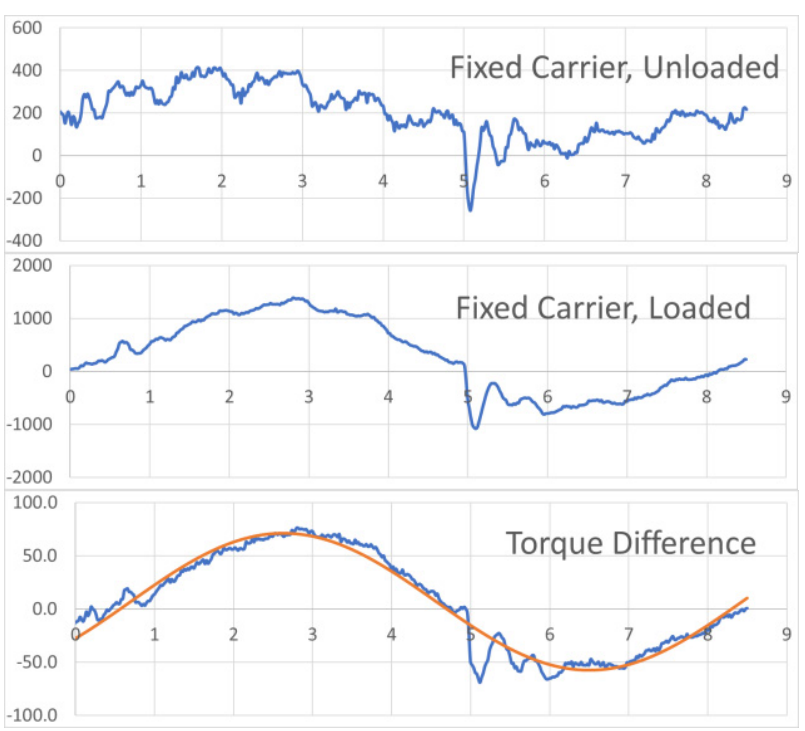

Fig. 8. Motor current (mA) drawn measured during a complete spin of the gearbox output $(8.3 \mathrm{sec}$.), under grounded carrier conditions, with (i) unloaded output (upper curve) and (ii) output loaded with a $0.5 \mathrm{~kg}$ mass, eccentrically positioned at a radius of $0.12 \mathrm{~m}$ (central curve). The curve below represents the motor torque difference $(\mathrm{mNm})$ calculated on the base on these measurements using the model given in (2), and represented together with its sinusoidal, fitting approximation (in red).

Assuming that the meshing efficiency of the two internal meshings are very close $(\eta \mathrm{i})$, with a grounded carrier the power flow inside the planetary gear train allows us to write:

$$
\eta_{c}=\eta_{i} \eta_{e} \approx 96.7 \%
$$

where $\eta e$ is the efficiency of the external meshing between the sun and each of the planets, which can be approximated assuming that the meshing losses are typically around two times those present in each of the internal meshings. This results in:

$$
\eta_{i} \approx 98.9 \% ; \eta_{e} \approx 97.8 \%
$$

\subsubsection{Fixed ring}

If we now ground the first ring of the Wolfrom gearbox (see blue "ring GND" element in Fig. 7) the system will operate with a gear ratio that corresponds to the following expression [26]:

$$
i=-\left(Z_{P 1} Z_{O} / Z_{S}\right)\left[\left(Z_{S}+Z_{R}\right) /\left(Z_{P 1} Z_{O}-Z_{R} Z_{P 2}\right)\right]=96.2
$$

Proceeding in an analog way as in subsection 3.2.1, Fig. 9 presents the resulting motor torque required to spin the $0.5 \mathrm{~kg}$ load in the specified test conditions, together with its sinusoidal, fitting approximation. 


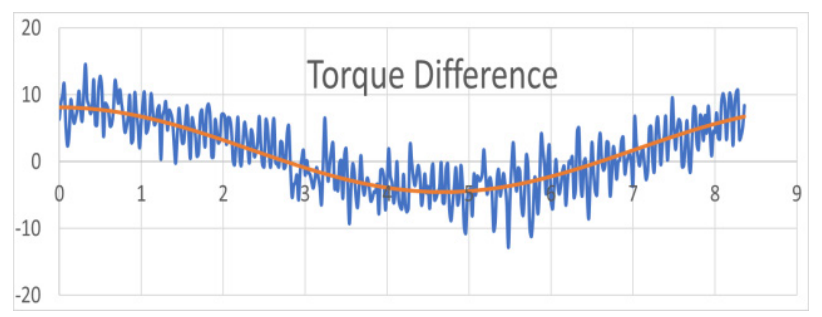

Fig. 9. Motor torque difference $(\mathrm{mNm})$ calculated on the base on the current measurements for loaded $(0.5 \mathrm{~kg}, 0.12 \mathrm{~m})$ and unloaded output with grounded ring, using the model given in (2), and represented together with its sinusoidal, fitting approximation (red).

Analog to what was done in subsection 3.2.1, the peak torque difference in this graph $(8.4 \mathrm{mNm})$ corresponds to a torque ratio of:

$$
t=\boldsymbol{\tau} \text { out } / \boldsymbol{\tau i n}=588 / 8.4=69.87
$$

This enables us to calculate the efficiency of the quasi-planetary configuration to spin the $0.5 \mathrm{~kg}$ mass at the peak torque point as:

$$
\eta r=69.87 / 96.2=72.6 \%
$$

Whereas, according to [26] the expected Wolfrom meshing efficiency, based on the efficiency values estimated in (7) should be:

$$
\eta^{\prime} r=(1 / i)\left[\left(1-i_{013} \eta_{e} \eta_{i}\right) /\left[\left(1-i_{053} \eta_{i} \eta_{i}\right]=79.2 \%\right.\right.
$$

where:

$$
\begin{gathered}
i_{013}=-Z_{R} / Z_{S}=7.5 \\
i_{053}=Z_{R} Z_{P 2} / Z_{O} Z_{P 1}=0.912
\end{gathered}
$$

The expected meshing efficiency $(79.2 \%)$ is therefore reasonably close to the measured efficiency value (72.6\%), especially considering that the addition of the $0.5 \mathrm{~kg}$ load also induces an increase in the loaddependent losses in the bearing elements, which is not taken into account in Eq. (11).

\section{Conclusion}

This paper demonstrates that the HRI actuation has different requirements than conventional industrial robotics. Reassessing the potential of planetary gear trains from an HRI perspective shows that Wolfrom configurations demonstrate an interesting potential. They are compact, can provide very high gear ratios, and their disc shape is advantageous for high torques.

The first experimental results with a concept prototype presented in this paper provide an initial validation of the ranges of gear gains and system efficiencies that can be attained.

To confirm the potential of this technology for HRI, our next step will be to test this technology in a lower limb prosthesis. For this, a new functional prototype will be designed for this specific application, and manufactured with conventional, serial means.
This research is partially funded by the FWO (Fonds voor Wetenschappelijk Onderzoek) Research Foundation Flanders, with personal FWO- grants of $\mathrm{T}$. Verstraten (postdoctoral fellowship), S. Crispel and E. Saerens (PhD fellowships).

\section{References}

1. M. Grebenstein, A. Albu-Schaeffer, S. Wolf et al.: The DLR hand arm system, 2011 IEEE Int. Conf. on Robotics and Automation: 3175 - 3182 (2011)

2. P. Polygerinos, Z. Wang, C.J. Walsh: Soft robotic glove for combined assistance and at-home rehabilitation, Robotics and Autonomous Systems 73: 135-143 (2015)

3. HH. Schempf, and DR. Yoerger: Study of Dominant Performance Characteristics in Robot Transmissions, ASME J. Mech. Des., 115 (3): $472-$ 482 (1993)

4. A.J. Veale, and S.Q. Xie: Towards compliant and wearable robotic orthoses: a review of current and emerging actuator technologies, Med. Eng. Phys., 38 (4): 317-325 (2016)

5. N., Kashiri, N. Tsagarakis, et al.: An overview on principles for energy efficient robot locomotion, Frontiers Robotics AI, 5 (DEC): 129 (2018)

6. J. W. Sensinger, and J. H. Lipsey: Cycloid vs. harmonic drives for use in high ratio, single stage robotic transmissions, 2012 IEEE Int. Conf. on Robotics and Automation, MN: 4130-4135 (2012)

7. P. Lopez Garcia, S. Crispel, D. Lefeber, et al.: Failure Mode end Effect Analysis (FMEA)-driven design of a planetary gearbox for active wearable robotics, $4^{\text {th }}$ Int. Symposium on Wearable Robotics: 460 (2018)

8. D. Torricelli, D. Lefeber, J.L. Pons, et al.: Humanlike compliant locomotion: state of the art of robotic implementations, Bioinspiration \& Biomimetics, 11: 051002 (2016)

9. T. Verstraten, B. Vanderborght, D. Lefeber, et al.: Modeling and design of geared DC motors for energy efficiency: Comparison between theory and experiments, Mechatronics, 30: 198-213 (2015).

10. J. W. Sensinger: Selecting motors for robots using biomimetic trajectories: optimum benchmarks, windings, and other considerations, 2010 IEEE Int. Conf. on Robotics and Automation, AL: 4175-4181 (2010)

11. R. Alo, F. Bottiglione, G. Mantriota: An innovative design of artificial knee joint actuator with energy recovery capabilities, Mechanisms and Robotics, 8 : 1310 (2014)

12. D. Dong, B. Convens, P. Cherelle, et al.: The effects of variable mechanical parameters on peak power and energy consumption of ankle-foot prostheses at different speeds, Advanced Robotics 32-23: 12291440 (2018)

13. B. Vanderborght, A. Albu-Schaeffer, A. Bicchi, et al: Variable impedance actuators: A review, 
Robotics and Autonomous Systems, 61-12: $1601-$ 1614 (2013)

14. Engineering Data CSD-2A Component Sets, catalog, Harmonic Drive AG, (2014)

15. A. Parmiggiani, G. Metta, et al.: On the performance of actuators with Harmonic Drive speed reducers. 14th Int. Conf. on New Actuators: 23-25 (2014)

16. M.R. Pitkin: Synthesis of a cycloidal mechanism of the prosthetic ankle, Prosthetics and Orthotics International, 20-3: 159-171 (1996)

17. H.W. Mueller: Die Umlaufgetriebe, Berlin, Springer-Verlag (1998)

18. A. Kapelevich: High Gear Ratio Epicyclic Drives Analysis, Gear Technology 06-2014: 62-67 (2014)

19. H.E. Merritt: Gear Trains, London, Issac Pitman \& Sons Ltd (1947)

20. B.R. Höhn, K. Stahl, P. Gwinner: Improved Efficiency for High-Ratio Planetary Gear Transmissions: Low-loss Wolfrom transmission for wind turbines. Getriebe Aktuell 2014 1-1: 6-11 (2014)

21. ANSI/AGMA 6123-C16, Design manual for enclosed epicyclic gear drives (2016)

22. K. Arnaudov, D. Karaivanov, L. Dimitrov: Some practical problems of distribution and equalization of internal loads in planetary gear trains, 4th International Conference on Power Transmissions (2012)

23. ANSI/AGMA 2001-D04, Fundamental rating factors and calculation methods for involute spur and helical gear teeth (2004)

24. A. Mihailidis, I. Nerantzis: A New System for Testing Gears Under Variable Torque and Speed, Recent Patents on Mec. Eng., 2-3: 179-192 (2009)

25. D.A. Winter: Biomechanical motor patterns in normal walking, Motor Behavior 15-4: 302-330 (1983)

26. J. Looman: Zahnradgetriebe: Grundlagen, Konstruktionen, Anwendungen in Fahrzeugen, Berlin, Heidelberg and New York, Springer-Verlag (1996) 